\title{
What Psychology Do Medical Students Need to Know? An Evidence Based Approach to Curriculum Development
}

Cordingley L. PhD, C Psychol. ${ }^{1}$

Peters S PhD, C Psychol. ${ }^{2}$.

Hart J. PhD. C Psychol. ${ }^{3}$.

Rock J. ${ }^{4}$

Hodges L. PhD. ${ }^{5}$.

Jean McKendrie $\mathrm{PhD}^{6}$

Bundy C. ${ }^{*}$ PhD, C Psychol. ${ }^{1 .}$

1. Dermatological Sciences, Institute for Inflammation \& Repair, University of Manchester and Manchester Academic Health Sciences Centre, Manchester UK.

2. Manchester Centre for Health Psychology, School of Psychological Sciences, University of Manchester and Manchester Academic Health Sciences Centre, Manchester UK.

3. Manchester Medical School, Manchester Centre for Health Psychology,

4. Keele Medical School, University of Keele,

5. University of Edinburgh,

6. Hull York Medical School

${ }^{*}$ corresponding author

Dr. Chris Bundy, 6.10 Williamson Building University of Manchester, Oxford Rd, Manchester M13 7PT UK

Indicate date CTA sent to the HEA:

Word count:3202

Type of Paper: practice paper.

\section{Keywords}

Behavioural and social sciences; medical education; curriculum development.

\section{Abstract:}

While the contribution of behavioural and social sciences for understanding health, illness and medical practice is made explicit in documents such as Tomorrow's Doctors, research shows that the proportion of curriculum space given to psychology in undergraduate curricula varies widely between medical schools. In the US, recommendations for behavioural sciences education for medical undergraduates have been developed. However, the United Kingdom has yet to produce agreed curriculum outcomes for behavioural sciences in medical education. 
We aimed to develop an evidence-based, consensus behavioural sciences curriculum for undergraduate medical education. This paper reports a novel technique for curriculum development that utilises knowledge and expertise of key stakeholders from medicine, medical education and behavioural sciences. It was successfully used to develop a psychology core curriculum for undergraduate medicine in the UK. 


\section{BACKGROUND}

Social and behavioural factors are important in the treatment and prevention of almost all the major diseases and nearly half of preventable deaths can be linked to behaviours ${ }^{1}$. Psychological factors such as beliefs and expectations also influence progress in long-term conditions (LTCs) and impact on health outcomes and a substantial body of literature has now accrued outlining the actual and potential pathways between psychological factors and disease end points. ${ }^{2-5}$

In addition, the practice of medicine is intellectually, emotionally and behaviourally demanding. Doctors experience higher levels of divorce, alcoholism and burnout compared with other professional groups ${ }^{6}$ and medical practitioners as a group demonstrate higher levels of stress compared with other professional groups ${ }^{7,8}$ and they tend to be overly self-critical as students which predicts later distress. ${ }^{9}$ These are not just problems for the practitioners themselves and their families but is a quality of care issue as stressed doctors are more prone to making errors. ${ }^{10}$

Despite longstanding arguments for the inclusion of Behavioural and Social Sciences

(B\&SS) in medical education ${ }^{11}$, the establishment of the first department of B\&SS in a North American medical school in $1959^{12}$ and the incorporation of B\&SS in assessment in the US in $1976^{13}$, in the UK systematic incorporation of B\&SS is a relatively recent occurrence and attempts to integrate with medicine have been met with varying degrees of success ${ }^{16}$. There is as yet no dedicated B\&SS department in a UK medical school and no consensus on what should be taught, or how it should be delivered, when, and by whom. 


\section{Developments within UK Medicine}

This is not to say that the potential contribution of B\&SS equipping medical students with knowledge that will both improve their own effectiveness with patients, and offer protection from 'burnout' by identifying and managing sources of stress, has not been acknowledged in the UK. The Royal Commission on Medical Education (the Todd Report, 1968) and General Medical Council's (GMC) Education Committee Working Party on the Teaching of the Behavioural Sciences, Community Medicine and General Practice in Basic Medical Education report in 1987, demonstrate respectively awareness of the need to improve B\&SS teaching for medical undergraduates and progress to the point where virtually every medical undergraduate curriculum had a well-developed syllabus in B\&SS (most of them concentrating upon psychology). ${ }^{17}$ Further developments followed the UK GMC recognition that undergraduate education in the UK was not fit for purpose over a decade ago. The result, a set of recommendations published as Tomorrow's Doctors $^{18}$ signalled a shift in emphasis away from the learner simply acquiring factually based information to the new graduate being able to apply the knowledge and skills acquired to medical practice. The primary recommendations were to reduce the factual content of programmes, contextualise the learning and make better use of modern teaching and learning methods. It was recommended that the B\&SS be included in the curriculum on a 'need to know' rather than 'nice to know' basis and strongly recommended that more emphasis be placed on learning to communicate better.

Further Tomorrow's Doctors revisions, 'Good Doctors, Safer patients' (CMO recommendations on national assessment) and Good Medical Practice ${ }^{20,21}$ reinforce the value of the B\&SS in teaching programme. Furthermore, psychological principles underpin a range of related topics such as patient safety, communication and patient 
centeredness. In fact, communication skills training has been the vehicle for delivery of much of the B\&SS curriculum in undergraduate education.

The current situation in the UK reflects local centres of excellence, a small number of departments that have well established expertise, but most medical schools show wide variability in the B\&SS curricular content; the teaching delivery methods and the learning outcomes are different; assessment strategies differ and the B\&SS in medical education remain largely invisible other than those components embedded in communication training. ${ }^{23}$ In addition, the degree of horizontal integration between specialities, differs between medical schools. Vertical integration (whether subject teaching spans the whole programme or is confined to the pre-clinical years) is also dependent on the institution. Finally, some staff delivering this component are not subject specialists; some may possess a first degree in a related discipline while others posses a related allied health qualification only. ${ }^{23}$ Of those who are B\&SS discipline experts, many quickly become dissatisfied and leave because they are poorly supported and there is an absence of career development pathways for those in post. ${ }^{24}$

Development of learning and teaching opportunities are also affected by a variety of attitudes and beliefs about B\&SS.

The conceptual and theoretical perspectives advanced in B\&SS may clash with those dominant in medicine ${ }^{26}$, the focus on exploratory and discursive techniques may be perceived as less useful than those that emphasise acquisition of facts and practice of skills. Senior role models may not value or endorse B\&SS perspectives and approaches. There may be hostility to perceived threats to medical autonomy and perceptions that B\&SS lack scientific legitimacy ${ }^{12,24}$. They may see it as valuable only as a contribution to systematic thinking, if it has application as a contributor to 
for example psychiatry, or as an adjunct to development of interpersonal skills relevant to medical consultations ${ }^{30}$. The status of B\&SS academics may be perceived to limit their contribution to medical education and they have been criticised for a lack of attention to application of their expert knowledge to medicine ${ }^{16,27,28}$. They may be perceived as too critical of medicine and unhelpful to students ${ }^{15,29}$. As a result students become critical and dismissive of B\&SS seeing the disciplines as subjective, not amenable to scientific study, and not having any evidence base other than "common sense". ${ }^{32}$ B\&SS may be perceived as nice to know but not need to know populated with concepts that are not familiar and require work if they are to derive the relevance to medical practice ${ }^{15}$

\section{Need to develop a core curriculum for the B\&SS in Medicine}

The GMC (Tomorrow's Doctors 209) recommends that graduates should “...apply psychological principles, method and knowledge to medical practice". ${ }^{33} \mathrm{p} 15$ In this study we focus on defining the relevant aspects of psychology that medical graduates need to know based on a rigorous and inclusive methodology, utilising the model for integrating B\&SS developed by US medical schools. ${ }^{22}$ In developing a core curriculum recommendation that was evidence-based, relevant to clinical practice and acceptable to stakeholders, a novel consultative methodology was employed.

\section{METHOD}

Three phases of work comprised data collection: 1) topic mapping; 2) topic prioritisation; and 3) data interpretation, integration and validation.

\section{Phase 1. Mapping psychology to medical education and practice}




\section{Procedure}

We sampled psychology topics taken from the British Psychological Society (BPS) minimum syllabus for graduate basis for registration with the BPS ${ }^{34}$ the BPS syllabus topic areas for postgraduate qualifications in Health Psychology and Occupational Psychology. ${ }^{35,36}$ These lists did not imply the depth or level of study specified in a core curriculum for medicine. A final list of 49 separate topics were identified in 7 areas (Box 1).

[Box 1 about here]

Participants were recruited at workshops conducted at two international medical education conferences (one in the US and one in the Netherlands) and worked in six mixed professional groups. At each workshop a 15 minute presentation was delivered to orientate participants to the sorting task. It covered the GMC's Good Medical Practice (GMP) framework and the concepts in the BPS Core Curriculum.

Individuals formed small mixed professional groups (6-8 participants) and were each given a set of cards that contained definitions and examples of the 49 psychology concepts and an A0 size map of the GMP categories (Figure 1). To make the sorting task more engaging, the cards were divided into four 'suits' using the familiar playing card symbols: 15 cognitive and developmental psychology topics (Clubs); 11 social psychology and individual differences topics (Spades); 9 Health and Clinical psychology topics (Hearts) and 14 Organizational Psychology topics (Diamonds).

Participants were instructed to discuss each topic as it arose from the deck and to place it on the category of GMP on the map. Blank cards were issued if people wanted to place one concept on more than one GMP category. If a concept could not 
be placed in any of the $7 \mathrm{GMC}$ categories it was recorded as not relevant.

Consensus was sought but if the group could not agree on a particular concept this was also recorded.

[Figure 1 about here]

The atmosphere created was congenial and encouraged discussion and interaction within the groups with a deliberate attempt to break down barriers between participants from different disciplinary/professional backgrounds. Organisers aimed to ensure that participants did not focus on defining jargon or too engrossed with detail or disagreement about what the GMP categories meant. The aim was to keep participants' thinking at the level of 'how could this psychology topic contribute to good medical practice?'. Each workshop took an hour to complete and the data on the categories and topics were recorded.

Results from Phase 1

Forty-seven participants took part in a workshop. Of these, $33(57.6 \%)$ were medical practitioners, 10 were psychologists (30.3\%), and the remaining 4 stated that they were medical educationalists (12.1\%). These formed six groups.

The vast majority of topics were viewed as relevant to Good Medical Practice with most cards placed in two or more of the GMP sections. Very few cards were placed only in 1 GMC category, with the majority placed in 2 or more (range $0-6$ from a possible total of 7). Only one topic was discarded by participants as not relevant. Specifics of psychology topics mapped to GMP sections are detailed in the core curriculum document. ${ }^{37}$ 


\section{Phase 2: Topic Prioritisation}

\section{Procedure}

A national meeting was arranged for thirty experts from the newly formed UK Psychology in Medicine network, all of whom were currently involved in medical education. Prior to the meeting, the topic list above was sent to delegates. A definition, one or two indicative key aspects of the topic and illustrative examples were provided alongside each topic (Figure 2). Apart from the topic that was unanimously viewed as irrelevant in Phase 1, all topics were included. Participants were asked to judge whether the topic should be high, medium, low or no priority, or whether it was an area for postgraduate medicine only. The priority levels are shown in Box 2.

[Box 2 and Figure 2 about here]

Documents were distributed in advance of the meeting to ensure they had sufficient time to consider the materials and make their priorities. During the meeting participants were allocated into three groups of five to six delegates each and asked to discuss the prioritisation task and topics. Each group recorded on a flipchart the points to emerge from the discussion and in turn reported these to the group as a whole.

\section{[Table 1 about here]}

\section{Results from Phase 2}

Detailed findings are provided in Table 1. The topic priorities reported are the modal option for the whole sample. Where no modal option appeared, the lower priority was given, thereby giving a conservative view of relevance. Group discussions during the 
event were recorded and that data were subjected to thematic analysis. The following main points were identified.

a) Relevance and context. Whilst most viewed the majority of areas as relevant, all participants stressed the need to contextualise psychology topics by embedding teaching activities within other disciplinary areas and with the patient experience. Some concern was expressed that a curriculum could simply be seen "as a checklist" and that medical schools would not "think out of the box". Curriculum integration was seen as having particular importance for social and behavioural sciences.

Additionally, it was recognised that the way the topics were presented needed to reflect the priorities of medical rather than psychology curricula. Thus new topic headings that highlighted patients' experiences, doctors' experiences and the learning process were elicited, debated and agreed.

b) Depth. The group emphasised the need for medical students to have sufficient knowledge of psychological principles that would allow them to apply this knowledge and recognise their use of them in practice. However, they were also clear that as medical practitioners they will be working as applied scientists but the depth of understanding need not mirror that of graduate psychology students.

c) Gaps in the topic list. Additional core topics (identified by at least three participants) included pain, mental illness, bereavement, body image and addiction. The large group discussion included substantial debate about the role of psychology in the professional and intellectual development of medics. Thus as well as core knowledge, other aspects of psychology were judged to be crucial to teaching, learning, assessment, and professional practice. 
d) Overlap with other disciplines. Participants emphasised the importance of the interfacing of psychology with key areas (e.g. public health, medical sociology, ethics, psychiatry). Topics such as poverty and service delivery were viewed as relevant but belonged in the domains of sociology or social policy rather than psychology and these were therefore omitted.

Readers recognised that research methods training was a particularly strong component of undergraduate psychology curricula but some were wary of presenting the view that this area is the sole remit of 'psychology'. There was disagreement as to whether 'research methods' was an area that should be taught by psychology experts. It was decided not to proclaim this area as solely the domain of psychology, but that it would be helpful for students to be aware of specific areas of expertise such as assessment of quality of life, attitudes and other complex constructs. This would complement other research methods and statistical analyses teaching in epidemiology.

\section{Phase 3: Data interpretation, integration and validation}

Topics selected as high priority from Phase 2 were organised as to which aspects of medicine, medical education and professional development the linked with. We aimed to embed the prioritised aspects of psychology with medical topics in order demonstrate integration and learning opportunities in the curricula.

The majority of topics were grouped under the heading of Psychology- core knowledge, with the remainder as Psychology for professional practice and Psychology - contribution to the educational process. The detailed output of this phase forms the curriculum document. ${ }^{36}$ Table 2 outlines key topic areas under each 
of these sections. Topics that were identified as more suitable for postgraduate level study are included in Table 3)

[Table 2 and 3 about here]

Validation: Critical readers, all experts in UK medical programmes and each with at least ten years involvement with undergraduate medicine delivery and all currently professorial appointments were asked to read the curriculum and comment on appropriateness, relevance and structure. They included heads of UK medical schools, Professors of medical education and Professors of psychology.

The process and content has been endorsed by six senior colleagues (see foreword to the core curriculum ${ }^{37}$ ), all of the written comments were positive and stressed the urgency for making the recommended curriculum available for use in designing/redesigning existing programmes. ASME, HEA subject centres and the BPS have also endorsed the core curriculum. In addition, the core curriculum was referenced in the latest GMC guidelines to medical schools. ${ }^{33}$

\section{DISCUSSION}

This paper describes the application of an expert consensus technique, an iterative process of consultation, participation and integration, to the development of an evidence based core curriculum in behavioural sciences for undergraduate medicine.

Each phase of the process required stakeholders to interrogate aspects of psychology and agree on how they could be applied to undergraduate medical 
education. The result is a curriculum that reflects current population health needs as viewed by medical educators and behavioural and social scientists.

A manageable and coherent curriculum, ${ }^{37}$ the outline of which appears here, can be applied to medical undergraduate programmes in the UK. This curriculum has the support of prominent psychologists, medical educationalists and practitioners and has been endorsed by the BPS and GMC Education committee.

Experts agreed the depth of coverage of particular areas should be limited to what newly graduating doctors require. Medical students should not be required to know details of theory development, rather they need to achieve sufficient understanding of a topic to inform their practice and decision making at this stage of their career.

Consideration of a number of issues is crucial in ensuring successful implementation of this core curriculum, these include:

Hidden curriculum. Only by understanding the barriers to why the B\&SS have not been incorporated into medical undergraduate programmes can any future attempts to implement this integrated core curriculum stand a better chance of succeeding. Implicit values are conveyed to students through the hidden curriculum ${ }^{38}$ It is likely the hidden curriculum undermines the perceived relevance of the B\&SS component of medical undergraduate programmes and changing that culture may be the biggest challenge to B\&SS experts in the next decade.

Establishing relevance is crucial, especially in the UK where medical students are younger and may enter with less developed understanding of the psychological aspects of health, illness and medical practice. Thus, integrated curricula may have greater value to non-graduate entry programmes and overcome some of the 
attitudinal barriers towards B\&SS in medical curricula. Recent work in the US has attempted to understand more about integration. ${ }^{39}$ The corollary of this, however, is that B\&SS experts have to be suitably equipped with a sound understanding of the practice of medicine and medical education.

\section{CONCLUSION}

This managed process of engaging both medical and psychology experts in a common, focused task resulted in consensus of what psychology is relevant to undergraduate medicine and may have contributed to the creation of more open and positive views of psychology in a medical curriculum. This process could facilitate the adoption of a more systematic and evidence-based curriculum for psychology in medical education and could be used by the other disciplines in medicine including; medical sociology and healthcare ethics and law. It may have relevance for development of B\&SS teaching in other healthcare professions.

\section{Recommendations}

We recommend the adoption of a core curriculum for psychology in medicine. A core curriculum in the UK has the following advantages:

1. Supporting medical schools to deliver key components of Tomorrow's Doctors. Medical practitioners and psychologists with expertise and experience in medical education have provided schools with detailed content and suggestions for integration and implementation.

2. Better standardisation of the students experiences of teaching and learning about the psychology contribution to medicine across UK medical schools. This 
encourages medical graduates to view psychology as making an important contribution to their practice, sending a signal to students that it is core rather than optional knowledge for practicing medicine.

3. Providing opportunities for quality control by internal assurance and external validation procedures. It would help to build a bank of expertise that could be used to evaluate programmes across the UK.

4. Providing opportunities for further development of competencies in the B\&SS by generating interest in developing a forum for sharing best practice and for facilitating research in order to facilitate good medical practice.

5. A core curriculum will support the professional identity and develop career pathways of subject experts who may be relatively isolated in medical schools. This would prevent the loss of such expertise from medicine to other areas of health care.

6. It could facilitate sharing assessment material across universities (for example via the UK medical schools national assessment partnership).

7. Finally, the most important outcome is the expectation that better knowledge and skill in the B\&SS domains will have an impact on patient health outcomes. 
Box 1: The basic areas of psychology used for the Phase 1 topic mapping task

Cognitive Psychology

Social Psychology

Developmental Psychology

Individual Differences

Research Methods

Health and Clinical Psychology

Occupational Psychology (how people remember, think, learn, perceive, attend to and manage information)

(how individuals think \& interact at individual, group and societal levels)

(acquisition and changes in psychological processes from conception to old age)

(factors accounting for differences between individuals, in particular intelligence and personality)

(quantitative \& qualitative design, analysis, ethics, governance and measurement)

(psychological processes that contribute to physical and mental health and illness)

(psychological processes involved in performance at work, in training, how organisations function).

Figure 1: Task mat used in mapping phase.

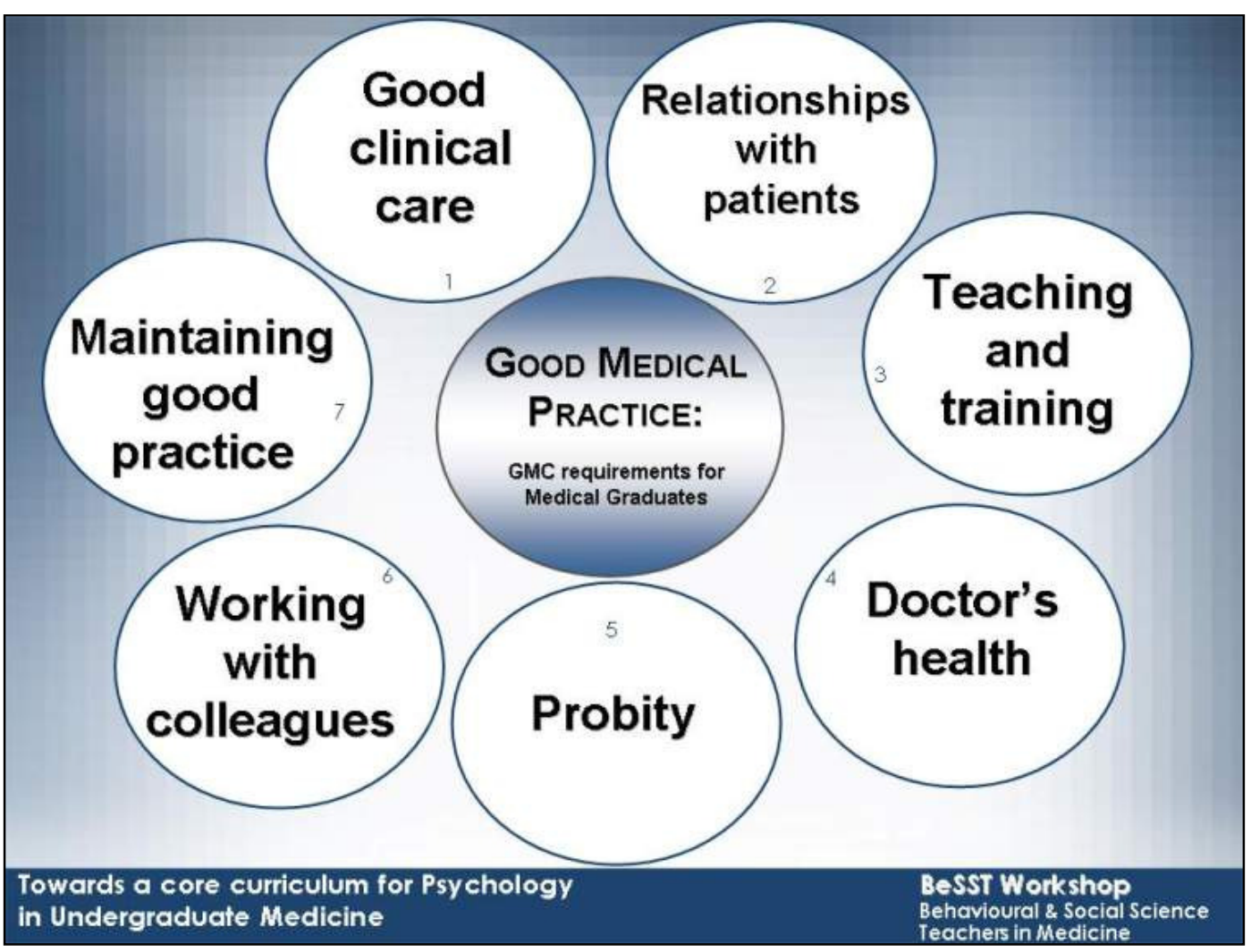


Box 2: Definition of priority levels used in Phase 2

High Priority: Core knowledge - undergraduate medical students should learn basic psychological concepts and application in medicine

Medium Priority: Core knowledge - undergraduate medical students should learn how the psychological concepts involved are applicable to medicine (but not the basic psychological concept)

Low Priority: Useful knowledge but not essential for all UK undergraduate medical programmes

Postgrad only: Not appropriate for an undergraduate curriculum but should be included in specialist postgraduate education or training

Not relevant: Not relevant to medical education or training

Figure 2: Topic layout used in prioritisation response task with example

\begin{tabular}{|c|c|c|}
\hline Topic: D3. & \multicolumn{2}{|c|}{$\begin{array}{l}\text { Definition: The changes as a function of age and experience } \\
\text { during later life. }\end{array}$} \\
\hline & & Topic Priority \\
\hline $\begin{array}{l}\text { Indicative Key } \\
\text { Points: }\end{array}$ & Examples: & \multirow{3}{*}{$\begin{array}{l}\text { High Priority } \\
\text { Medium Priority } \square \\
\text { Low Priority } \square \\
\text { Not relevant } \square \\
\text { Postgrad only } \quad \square\end{array}$} \\
\hline - Normal & $\begin{array}{l}\text { Ability to differentiate normal } \\
\text { changes in cognitive function from } \\
\text { those caused by disease. }\end{array}$ & \\
\hline $\begin{array}{l}\text { - Relationships } \\
\text { over the lifespan }\end{array}$ & $\begin{array}{l}\text { Role of social support in illness } \\
\text { prevention in old age. }\end{array}$ & \\
\hline
\end{tabular}


Table 1 Findings from Phase 2 - Psychology Core Knowledge with prioritisation $\underline{\text { levels }}$

1. Psychological factors in health and illness.

Psychological factors in health promotion and

Topic illness prevention

Psychological Interventions

Psychological processes in disease

Pain

Genes and Behaviour

Mental Health and Mental IIIness

Priority

High

High

High

High

High

High

2 Psychological responses to illness

Emotional, cognitive and behavioural responses to illness

Coping with illness

High

High

3 Psychology across the life span

Cognitive development

Cognitive aspects of ageing

Social development across the lifespan

Death, dying and bereavement

Assessment of cognitive function over the age span

Attachment

High

High

High

High

Medium

Medium

4 Cognitive functioning in health and illness.

Memory

Learning

Sleep and consciousness

Attention

Perception

Language

High

High

High

Medium

High

High

$5 \quad$ Psychology for professional practice

Clinical reasoning and decision making

High

Human communication and communication skills

High

training

Research methods and evidence based

Medium

medicine

Social processes shaping professional behaviour

Stress, well-being and burn-out

High

Leadership and team-working

High

High

Teaching the next generation of doctors

High 
Table 2: Integrated curriculum summary

\section{Psychology - core knowledge}

1.1 Psychological factors in health and illness

1.2 Psychological responses to illness

1.3 Psychology across the life span

1.4 Cognitive functioning in health and illness

\section{Psychology for professional practice}

2.1 Clinical reasoning and decision making

2.2 Human communication and communication skills training

2.3 Research methods and evidence-based medicine

2.4 Social processes shaping professional behaviour

2.5 Stress, well-being and burn-out

2.6 Leadership and team-working

2.7 Teaching the next generation of doctors

\section{Psychology - contribution to the educational process}

\subsection{Learning to learn}

3.2 Skills training

3.3 Reflective practice

3.4 Situated learning

3.5 Feedback and appraisal

3.6 Assessment design and quality assurance

\section{Psychology topics - Post graduate level only}
4.1 Leadership
4.2 Selection and Appraisal
4.3 Organisational Change

Table 3. Topics identified as Post graduate level only

\begin{tabular}{|l|l|}
\hline $\begin{array}{l}\text { Leadership - The exercise of influence over a group or } \\
\text { individual to achieve certain goals. from }\end{array}$ & $\begin{array}{l}\text { Organisational } \\
\text { Psychology }\end{array}$ \\
\hline $\begin{array}{l}\text { Selection \& Appraisal - The use of psychological methods to } \\
\text { inform selection and appraisal processes. }\end{array}$ & $\begin{array}{l}\text { Organisational } \\
\text { Psychology }\end{array}$ \\
\hline Organisational Change - Managing change processes. & $\begin{array}{l}\text { Organisational } \\
\text { Psychology }\end{array}$ \\
\hline
\end{tabular}




\section{REFERENCES}

1. McGinnis J, Foege W. Actual causes of death in the US. JAMA 1993;270 (18):2207-2212.

2. Andersen BL. Biobehavioural outcomes following psychological interventions for cancer patients. Journal of Consulting and Clinical Psychology 2002;70 (3):590-610.

3. Smith T, Ruiz J. Psychosocial influences on the development and course of coronary heart disease: current status and implications for research and practice. Journal of Consulting \& Clinical Psychology 2002;70 (3):548-568.

4. Barefoot J, Brummett B, Helms M. Depressive symptoms and survival of patients with coronary artery disease. Psychosomatic Medicine 2000;62 (6):790-795.

5. Kawachi I, Colditz GA, Ascherio A, Rimm EB, Giovannucci E, Stampfer MJ, et al. A prospective study of social networks in relation to total mortality and cardiovascular disease in men in the USA. J Epidemiol Community Health 1996;50 (3):245-251.

6. Vaillant G, Sobowale N, McArthur C. Some psychological vulnerabilities of physicians. New England Journal of Medicine 1972;287:372-375.

7. Firth-Cozens J. Doctors, their wellbeing, and their stress. British Medical Journal 2003;326 (7391):670-671.

8. Taylor C, Graham J, Potts HWW, Richards MA, Ramirez AJ. Changes in mental health of UK hospital consultants since the mid-1990s. The Lancet 2005;366 (9487):742-744. 
9. Firth-Cozens J. The role of early family experiences in the perception of organizational stress: fusing clinical and organizational perspectives. Journal of Occupational and Organizational Psychology 1992;65: 61-75.

10. Jones JW, Barge BN, Fay LM, Kunz LK, Wuebker LJ, Steffy BD. Stress and Medical Malpractice: Organizational Risk Assessment and Intervention. Journal of Applied Psychology 1988;73(4):727-735.

11. Flexner A. Medical education in the United States and Canada. A report to the Carnegie Foundation for the Advancement of Teaching. Boston, MA: Updyke, 1910.

12. Dacey A, Weintrob J. Human behaviour: the teaching of social and behavioural sciences in medical schools. Social Science and Medicine 1973;7:943-957.

13. Wexler M. The behavioural sciences in medical education. American Psychologist 1976:275-283.

14. WHO. The social sciences in medical education. WHO Chronicles 1970;24:478.

15. Tait I. Behavioural science in medical education and clinical practice. Social Science and Medicine 1973;7:1003-1011.

16. Benbassat J, Baumal R, Borkan JM, Ber R. Overcoming barriers to teaching the behavioural and social sciences to medical students. Academic Medicine 2003; 78:372-380.

17. GMC. Education Committee. The teaching of behavioural sciences, community medicine and general practice in basic medical education. London: General Medical Council; 1987.

18. GMC. Tomorrow's Doctors. Recommendations on undergraduate medical education. London: General Medical Council, 1993.

19. GMC. Tomorrow's Doctors. Recommendations on undergraduate medical education. London: General Medical Council. 2003 
20. GMC. Good Medical Practice. The Duties of a Doctor Registered with the GMC. London: General Medical Council. 2006

21. GMC. Good Medical Practice. The Duties of a Doctor Registered with the GMC. London: General Medical Council. 2001.

22. Cuff $P$, Vanselow N. Improving medical education: Enhancing the behavioral and social science content of medical school curricula. Washington, DC: National Academies Press, 2004.

23. Russell, A.J., E. van Teijlingen, H. Lambert \& R. Stacy. Social and Behavioural Science Education in UK Medical Schools: Current Practice and Future Directions. Medical Education 2004; 38 (4): 409-417

24. Litva A, Peters S. Exploring barriers to teaching behavioural and social sciences in medical education. Medical Education 2008;42 (3):309-314.

25. Boelen C. A new paradigm for medical schools a century after Flexner's report. Bulletin of the World Health Organisation 2002; 80 (7). WHO: Geneva.

26. Jaco EG. Problems and prospects of the social sciences in medical education. Journal of Health and Human Behavior 1960; 1: 29.

27. Bolman WM. The place of behavioural science in medical education and practice. Academic Medicine 1995; 70(10): 873-878.

28. Dacey A, Weintrob J. Human behaviour: the teaching of social and behavioural sciences in medical schools. Social Science \& Medicine 1973; 7: 943-957.

29. Peters S, Litva A. Relevant behavioural and social science for medical undergraduates: a comparison of specialist and non-specialist educators. Medical Education 2006; 40(10): 1020-1026.

30. Sheldrake P. Behavioural science in the medical curriculum. Social Science and Medicine 1973; 7: 967. 
31. Knight LV. A silly expression: Consultants' implicit and explicit understanding of Medical Humanities. A qualitative analysis. Med Humanities 2006;32:119-124.

32. Rees CE, Sheard CE, McPherson AC. (2002) A qualitative study to explore undergraduate medical students' attitudes towards communication skills learning. Med Teach. 2002;24(3):289-93.

33. GMC.Tomorrow's Doctors 2009 Tomorrow's Doctors. Outcomes and Standards for Undergraduate Medical Education. London: General Medical Council.

34. The British Psychological Society (BPS), Membership and Qualifications Board, Revised Syllabus for the Qualifying Examination 2004. BPS publications.

35. The British Psychological Society (BPS) http://www.bps.org.uk/networkscommunities/member-networks/divisions/division-healthpsychology/division-health-psychology

36. The British Psychological Society (BPS) http://www.bps.org.uk/networkscommunities/member-networks/divisions/division-occupationalpsychology/division-occupationa

\section{Removed to anonymise submission. Details included in full version}

38. Hafferty F. Beyond curriculum reform: confronting medicine's hidden curriculum. Academic Medicine 1998;73(4):403-7.

39. Satterfield JM, Adler SR, Chen HC, Hauer KE, Saba GW \& Salazar R. Creating an ideal social and behavioural sciences curriculum for medical students Medical Education 2010; 44: 1194-1202 